\title{
Phononic canonical quasicrystalline waveguides
}

Cite as: Appl. Phys. Lett. 116, 241903 (2020); https://doi.org/10.1063/5.0013528

Submitted: 12 May 2020 . Accepted: 04 June 2020. Published Online: 17 June 2020

M. Gei (D) Z. Chen, F. Bosi (D), and L. Morini

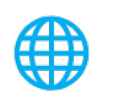

View Online

\section{ARTICLES YOU MAY BE INTERESTED IN}

Wave-canceling acoustic metarod architected with single material building blocks Applied Physics Letters 116, 241904 (2020); https://doi.org/10.1063/5.0011319

Large and continuous tuning of the work function of indium tin oxide using simple mixing of self-assembled monolayers

Applied Physics Letters 116, 241603 (2020); https://doi.org/10.1063/5.0005517

Gradient-index phononic crystals for omnidirectional acoustic wave focusing and energy harvesting

Applied Physics Letters 116, 234101 (2020); https://doi.org/10.1063/5.0008791

\section{Lock-in Amplifiers up to $600 \mathrm{MHz}$}
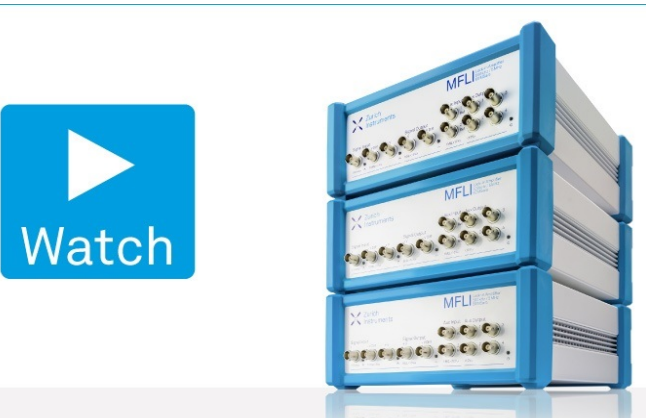


\title{
Phononic canonical quasicrystalline waveguides
}

Cite as: Appl. Phys. Lett. 116, 241903 (2020); doi: 10.1063/5.0013528

Submitted: 12 May 2020 • Accepted: 4 June 2020 .

Published Online: 17 June 2020

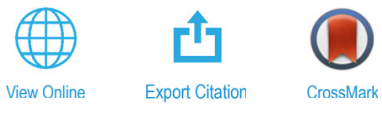

M. Gei, ${ }^{1,2, a)}$ (D) Z. Chen, ${ }^{1}$ F. Bosi, ${ }^{3}$ (D) and L. Morini ${ }^{1}$

\author{
AFFILIATIONS \\ ${ }^{7}$ School of Engineering, Cardiff University, Cardiff CF24 3AA, United Kingdom \\ ${ }^{2}$ DICAM, University of Trento, via Mesiano 77, 38123 Trento, Italy \\ ${ }^{3}$ Department of Mechanical Engineering, University College London, London WC1E 7JE, United Kingdom
}

${ }^{a)}$ Author to whom correspondence should be addressed: geim@cardiff.ac.uk

\begin{abstract}
The dynamic behavior of the class of periodic waveguides whose unit cells are generated through a quasicrystalline sequence can be interpreted geometrically in terms of a trace map that embodies the recursive rule obeyed by traces of the transmission matrices. We introduce the concept of canonical quasicrystalline waveguides, for which the orbits predicted by the trace map at specific frequencies, called canonical frequencies, are periodic. In particular, there exist three families of canonical waveguides. The theory reveals that for those (i) the frequency spectra are periodic and the periodicity depends on the canonical frequencies, (ii) a set of multiple periodic orbits exists at frequencies that differ from the canonical ones, and (iii) perturbation of the periodic orbit and linearization of the trace map define a scaling parameter, linked to the golden ratio, which governs the self-similar structure of the spectra. The periodicity of the waveguide responses is experimentally verified on finite specimens composed of selected canonical unit cells.
\end{abstract}

Published under license by AIP Publishing. https://doi.org/10.1063/5.0013528

The band structure of phononic spectra of periodic mechanical metamaterials has been extensively investigated in the last 40 years. ${ }^{1-4}$ Theoretical and experimental studies have shown how to take advantage of its properties to control several dynamical phenomena such as negative refraction of elastic waves, ${ }^{5-7}$ topological edge modes, ${ }^{8-10}$ wave focusing, ${ }^{11,12}$ mode conversion, ${ }^{13}$ and gap tunability. ${ }^{14,15}$ Recently, these analyses have been extended to inspect the dispersive properties of quasiperiodic and quasicrystalline phononic media. ${ }^{16-19}$ These are characterized by distinctive self-similarity features which can be explored to extend the range of capabilities of metamaterials. A powerful tool to examine the properties of dynamic spectra of uniaxial quasicrystalline-generated periodic waveguides is the trace map which defines, at each frequency, a discrete orbit on the corresponding invariant surface. ${ }^{20-25}$ For the class of quasicrystalline sequences coinciding with the Fibonacci chain, we show in this Letter that some configurations of the waveguide allow the orbit to be periodic at some particular frequencies and the periodicity is reflected on the whole spectra. Moreover, additional periodic orbits exist at frequencies where the invariant vanishes and the scaling of the self-similar structure of the spectra in the neighborhood of those frequencies can be quantitatively defined in terms of the golden ratio by linearizing the trace map.

We investigate a set of infinite two-phase structured rod whose elementary cells are generated by adopting the standard Fibonacci sequence. This is a particular case of the class of generalized Fibonacci sequences that follow the recursion $\operatorname{rule}^{26} \mathcal{F}_{i}=\mathcal{F}_{i-1}^{m} \mathcal{F}_{i-2}(i \geq 2)$, with $\mathcal{F}_{0}=S, \mathcal{F}_{1}=L$ setting the initial conditions in terms of the two basic constituents $L$ and $S$ (the natural exponent $m$ implies repetition of the base $m$ times). All waveguides designed from elements of this class share the same properties that are, therefore, illustrated with reference to the standard sequence that corresponds to $m=1 .^{27}$ Figure 1 shows two configurations where the lengths of the two segments $L$ and $S$ are indicated with $l_{L}$ and $l_{S}$, while $\alpha_{j}$ and $\rho_{j}(j \in\{L, S\})$ denote axial stiffness and mass density per unit length of each element, respectively. For any cell $\mathcal{F}_{i}{ }^{28}$ the Floquet-Bloch dispersion diagram for axial waves is governed uniquely by the trace of the transmission matrix $T_{i}(\omega)$, i.e., $x_{i}(\omega)=\operatorname{tr} T_{i}(\omega)$, through the equation $K(\omega)=\arccos \left(x_{i}(\omega) / 2\right),{ }^{29}$ where $K(\omega)$, the dimensionless Bloch number, is a real quantity if

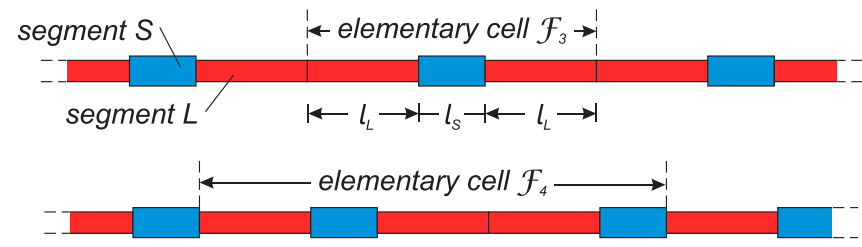

FIG. 1. Schematic of infinite quasicrystalline-generated canonical waveguides; sequences $\mathcal{F}_{3}$ and $\mathcal{F}_{4}$ are displayed. 
$\left|x_{i}(\omega)\right| \leq 2 .^{30,35}$ Due to the unimodularity of $T_{i}(\omega)$, i.e., $\operatorname{det} T_{i}(\omega)=1$, traces satisfy the recursive rule ${ }^{31}$

$$
x_{i+1}=x_{i-1} x_{i}-x_{i-2} \quad(i \geq 2),
$$

with initial conditions given by

$$
\begin{gathered}
x_{0}=2 \cos \left(\gamma_{S} \omega\right), \quad x_{1}=2 \cos \left(\gamma_{L} \omega\right), \\
x_{2}=2 \cos \left(\gamma_{S} \omega\right) \cos \left(\gamma_{L} \omega\right)-\beta \sin \left(\gamma_{S} \omega\right) \sin \left(\gamma_{L} \omega\right) .
\end{gathered}
$$

The impedance mismatch $\beta$ corresponds to $\beta=\left(\alpha_{L}^{2} Q_{L}+\alpha_{S}^{2} Q_{S}\right) /$ $\left(\alpha_{S} \alpha_{L} \sqrt{Q_{S} Q_{L}}\right)$, with $Q_{j}=\rho_{j} / \alpha_{j}$ and $\gamma_{j}=\sqrt{Q_{j}} l_{j}$. For $\beta=2$, the waveguide behaves as a homogeneous medium.

A new set of variables $\tilde{x}_{i}=x_{i+2}, \tilde{y}_{i}=x_{i+1}, \tilde{z}_{i}=x_{i}$ may be defined singling out the triplet $R_{i}=\left(\tilde{x}_{i}, \tilde{y}_{i}, \tilde{z}_{i}\right)$. Exploitation of (1) leads to the definition to the following trace map $\mathcal{T}$ determining the evolution of $R_{i}$ :

$$
R_{i+1}=\mathcal{T}\left(R_{i}\right)=\left(\tilde{x}_{i} \tilde{y}_{i}-\tilde{z}_{i}, \tilde{x}_{i}, \tilde{y}_{i}\right),
$$

through which it is shown that the quantity

$$
I(\omega)=\tilde{x}_{i}^{2}+\tilde{y}_{i}^{2}+\tilde{z}_{i}^{2}-\tilde{x}_{i} \tilde{y}_{i} \tilde{z}_{i}-4=\left(\beta^{2}-4\right) \sin ^{2}\left(\gamma_{S} \omega\right) \sin ^{2}\left(\gamma_{L} \omega\right)
$$

is an invariant. In the three-dimensional space spanned by the orthogonal co-ordinate system $O \tilde{x} \tilde{y} \tilde{z}$,

$$
\tilde{x}^{2}+\tilde{y}^{2}+\tilde{z}^{2}-\tilde{x} \tilde{y} \tilde{z}-4=I(\omega)
$$

represents the invariant surface which possesses six saddle points $P_{k}(k=1, \ldots, 6)$, opposite in pairs. ${ }^{32,33}$ Points $R_{i}$ obtained by iterating map (3) are all confined to this surface. Consequently, for any given frequency $\omega$, all points of the orbit generated through (3) can be mapped onto the surface defined by Eq. (5). According to the property of $\mathcal{T}$ and to the initial point taken as its argument, orbits can be either $p$-point periodic or non-periodic. In the former case, the discrete trajectory repeats itself after $p$ applications of $\mathcal{T}$, i.e., $\mathcal{T}^{p}\left(R_{i}\right)=R_{i}$.

A close inspection shows that saddle points $P_{k}{ }^{34}$ are all part of a 6-Point Periodic Orbit (PPO), namely, $\mathcal{T}^{6}\left(P_{k}\right)=P_{k}$; therefore, the question naturally arises: which type of elementary cell can be represented by such a close orbit on the surface (5)? The answer is that at a certain frequency, one of the following three conditions must be satisfied:

$$
\text { (1) } \tilde{y}_{0}=\tilde{z}_{0}=0, \quad \text { (2) } \tilde{x}_{0}=\tilde{z}_{0}=0, \text { (3) } \tilde{x}_{0}=\tilde{y}_{0}=0 .
$$

These requirements can be observed only for a particular set of configurations, called here the class of canonical sequences, and at particular values of the frequency, denoted as canonical frequencies. The three conditions (6) imply that two of the traces (2) vanish, and then, substituting them into (6), the following relationships are derived:

$$
C^{(1)}=\frac{1+2 j}{1+2 k}, \quad C^{(2)}=\frac{1+2 j}{2 q}, \quad C^{(3)}=\frac{2 q}{1+2 k},
$$

respectively, where $C=\gamma_{S} / \gamma_{L}$ and $j, k, q \in \mathbb{N}$. The superscript simply indicates the number of the condition concerned in (6) that will define the corresponding $r$ th family $(r=1,2,3)$ of canonical configurations. In formulas (7), it is important to remark that indices $j, k$, and $q$ are such that fractions on the right-hand sides are in lowest terms. We note that family no. 1 encompasses odd/odd ratios, while odd/even and even/odd ratios are associated with family nos. 2 and 3, respectively. The canonical frequencies for each family can be written as $\omega_{c n}^{(r)}$ $=\omega_{c}^{(r)}(1+2 n)(n \in \mathbb{N})$, where

$$
\omega_{c}^{(1)}=\omega_{c}^{(3)}=\frac{\pi}{2 \gamma_{L}}(1+2 k), \quad \omega_{c}^{(2)}=\frac{\pi}{\gamma_{L}} q .
$$

All conditions (6) force the functions $x_{i}(\omega)$ for canonical rods to be periodic, a property that leads to periodic stop- and pass-band (SPB) layouts. By defining as period of traces the least frequency range $\left[0, \omega_{t}\right]$ such that $x_{i}\left(\omega_{t}\right)=2$, it turns out that $\omega_{t}=4 \omega_{c}$. However, as $\operatorname{Im}(\mathrm{K})=0 \Longleftrightarrow\left|x_{\mathrm{i}}\right| \leq 2$, the period of both the frequency spectra and the invariant $I(\omega)$ is $2 \omega_{c}$.

(a)

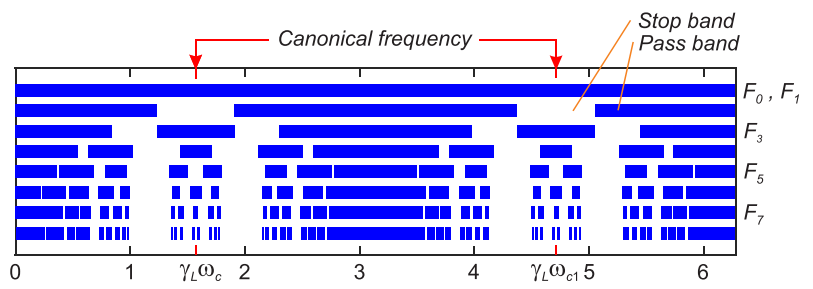

(b)

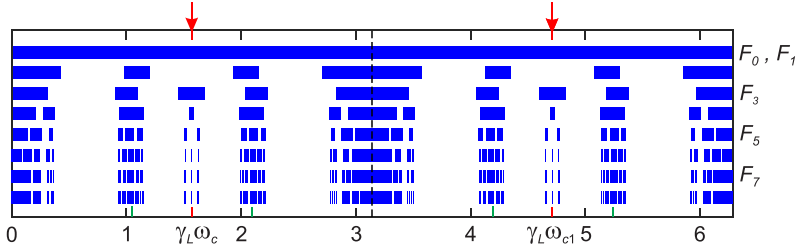

(c)

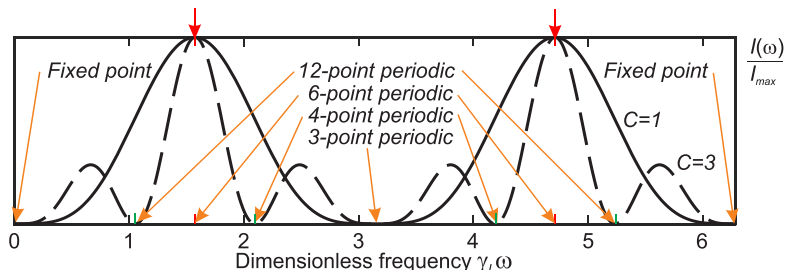

(d)

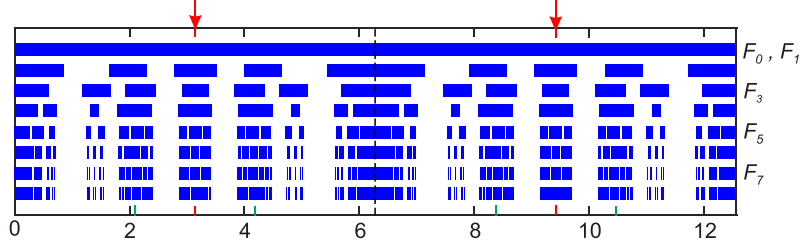

(e)

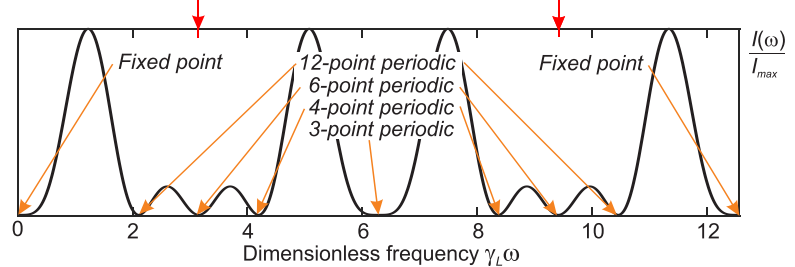

FIG. 2. Stop- (white)/pass- (blue) band (SPB) diagrams and function $I(\omega)$ for the first eight sequences of three representative canonical rods within the dimensionless period of traces $\left[0, \gamma_{L} \omega_{t}\right]$. (a) SPB diagrams for $C=1$ and (b) for $C=3$, (c) functions $I(\omega)$ for $C=1,3$, (d) SPB diagram, and (e) function $I(\omega)$ for $C=3 / 2$. $\omega_{c}$ and $\omega_{c 1}$ denote canonical frequencies, highlighted with red arrows. Frequencies at which periodic orbits occur are indicated in (c) and (e). 

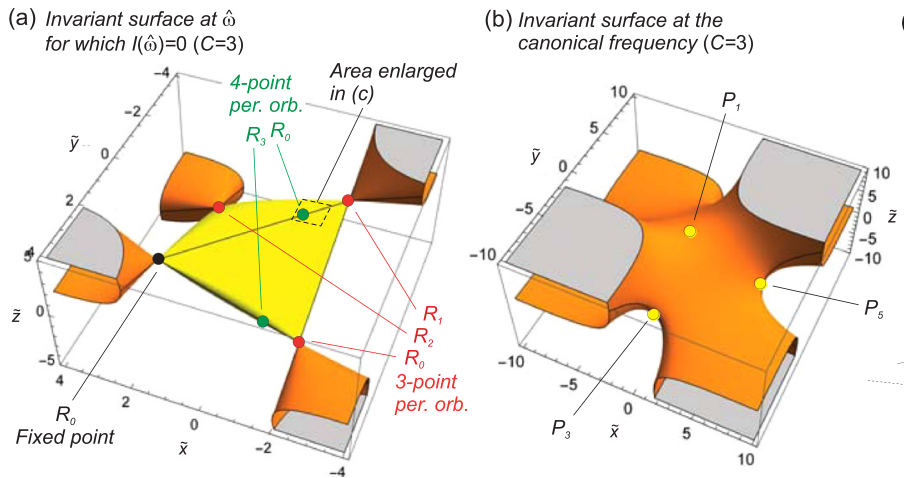

(c) Close-up view of the neighbourhood of point $R_{0}$

FIG. 3. Plots of the invariant surface for $C=3$ (a) for frequencies $\hat{\omega}$ : a 4-PPO $\left(\omega=\omega_{t} / 3\right)$ (green points), a 3-PPO $\left(\omega=\omega_{t} / 2\right)$ (red points), and a fixed point $\left(\omega=\omega_{t}\right)$ (black) are displayed; (b) for canonical frequencies $\omega_{c n}$ where three points of the 6-PPO are displayed. (c) Close-up view of the neighborhood of $R_{0}$ of the 4-PPO shown in (a) where the corresponding points $\bar{R}_{0}, \bar{R}_{4}, \bar{R}_{8}$ at the perturbed frequency $\bar{\omega}=\omega_{t} / 3+\delta \omega$ (with $\gamma_{L} \delta \omega=0.001$ ) are reported. $\bar{R}_{4}$ and $\tilde{R}_{8}$ are the predictions of the linearization of the trace map. $g$ represents the normalized unit eigenvector associated with the maximum eigenvalues $\kappa$.

The periodic SPB layout up to $\mathcal{F}_{8}$ and the plot of $I(\omega)$ of three representative canonical waveguides in the dimensionless range $\left[0, \gamma_{L} \omega_{t}\right]$, namely, $C^{(1)}=1,3$ and $C^{(2)}=3 / 2$, are reported in Fig. 2 where canonical frequencies are indicated by red arrows. The SPB spectra appear at a first glance periodic with a period of $\omega_{t} / 2$; however, a close inspection of transformation (3), performed in the ensuing paragraph, shows that the period is still $\omega_{t}$ and the diagrams are symmetric with respect to $\omega_{t} / 2$.

In addition to that singled out by the saddle points, a variety of periodic orbits on the invariant surface are present, located at frequencies $\hat{\omega}$ for which $I(\hat{\omega})=0$. To help illustrate some of the cases, in Fig. 3 (a) the invariant surface (5) for $I(\hat{\omega})=0 \quad(C=3)$ is sketched. Inspection of the expression of the invariant shows that $\forall C$ a fixed point $R_{i}=(2,2,2)$ (i.e., $\left.\mathcal{T}\left(R_{i}\right)=R_{i}\right)$ exists at $\omega_{t}$ [black point in Fig. 3(a)], whereas a 3-PPO is achieved at $\omega_{t} / 2$ [the three points are marked in red in Fig. 3(a)]. Additional possibilities arise depending on C. As an example, in Figs. 2(b) and 2(d), a 4-PPO is detected at both $\omega_{t} / 3$ and $2 \omega_{t} / 3$ and two out of the four points of the orbit are depicted in green in Fig. 3(a) for the former frequency. Other cases displayed in Fig. 2 and a general method to establish the existence of periodic orbits is described in the supplementary material.

The SPB diagram in the neighborhood of a frequency at which a periodic orbit takes place displays a self-similar pattern controlled by a scaling factor that is revealed by perturbing the corresponding periodic orbit and linearizing map (3). ${ }^{32}$ An example of perturbation is illustrated in Fig. 3(c) $(C=3)$, where the $4-\mathrm{PPO}$ at $\omega_{t} / 3$ is slightly changed by studying the open trajectory at $\bar{\omega}=\omega_{t} / 3+\delta \omega$ composed of points $\bar{R}_{i}=R_{i}(\bar{\omega})$, with $\gamma_{L} \delta \omega=0.001$. In summary (see the supplementary material), by denoting $\delta \boldsymbol{r}_{i}=\bar{R}_{i}-R_{i}$, it turns out that $\delta \boldsymbol{r}_{i+p v}$ $\approx \kappa^{v} \xi \boldsymbol{g}(v \in \mathbb{N})$, where $\kappa$ is the maximum eigenvalue of the matrix governing the linearized problem and $\xi \boldsymbol{g}$ is the component of $\delta \boldsymbol{r}$ along the normalized eigenvector $\boldsymbol{g}$ associated with $\kappa^{25}$ The golden ratio $\phi$ is the key parameter for scaling at $\hat{\omega}$ as $\kappa$ corresponds to $\phi^{3}, \phi,-\phi^{4}$ for the 3-PPO, the fixed point, and the 4-PPO, respectively.

In Fig. $3(\mathrm{c})$, red points mark the exact position of $\bar{R}_{0}, \bar{R}_{4}, \bar{R}_{8}$, while black points $\tilde{R}_{0}, \tilde{R}_{4}, \tilde{R}_{8}$ represent the corresponding placements provided by the linearization. The former are, by construction, aligned and belong to the tangent plane at $R_{0}$, whose equation is $\tilde{z}=2$. In particular, with $\delta \tilde{\boldsymbol{r}}_{0}=\xi \boldsymbol{g}, \tilde{R}_{0}=R_{0}+\delta \tilde{\boldsymbol{r}}_{0}, \quad \tilde{R}_{4}=R_{0}-\phi^{4} \delta \tilde{\boldsymbol{r}}_{0}, \quad \tilde{R}_{8}$ $=R_{0}+\phi^{8} \delta \tilde{\boldsymbol{r}}_{0}$.

Figure 4 illustrates some representative cases of scaling of selfsimilar SPB diagrams in the neighborhood of frequencies where

(a)
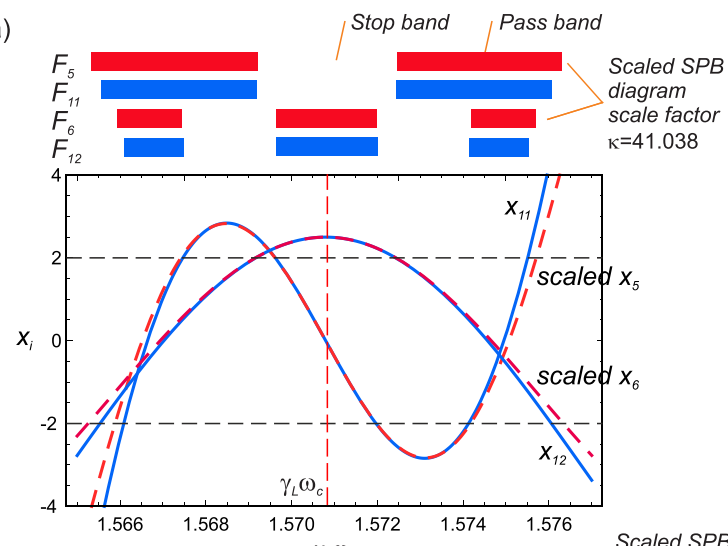

(b)
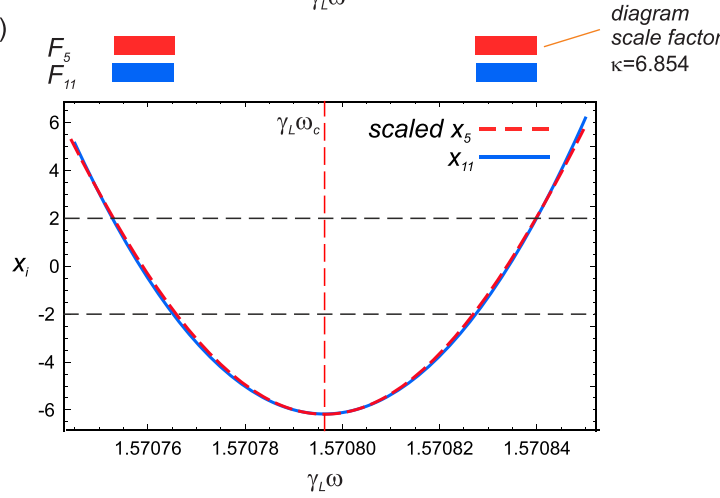

FIG. 4. Scaling of the self-similar structure of the SPB layout about frequencies where periodic orbits take place. (a) Plot of traces $x_{11}(\omega)$ and $x_{12}(\omega)$, scaled traces $x_{5}(\kappa \omega)$ and $x_{6}(\kappa \omega)$, and local SPB layout in the neighborhood of $\omega_{c}$ for $C=1$. (b) Plot of trace $x_{11}(\omega)$, scaled trace $x_{5}(\kappa \omega)$, and local SPB layout for $\mathrm{C}=3$ in the neighborhood of $\omega_{c}$. 
periodic orbits occur. Figure 4(a) is related to the canonical frequency $\gamma_{L} \omega_{c}=\pi / 2$ for $C=1$, where the orbit is composed of six points and the scaling factor is $\kappa=41.038$. It is shown that the scaled traces $x_{5}(\kappa \omega)$ and $x_{6}(\kappa \omega)$ are almost coincident with $x_{11}(\omega)$ and $x_{12}(\omega)$, respectively, and the corresponding scaled SPB layouts match almost exactly. Similar observations apply, at the same dimensionless frequency, to Fig. 4(b) to the pair $x_{5}(\kappa \omega)$ and $x_{11}(\omega)$ for $C=3$. Here, $\kappa=6.854$.

The periodicity of the dynamic spectra and the scaling of the SPB diagram can be also observed in canonical waveguides composed of an arbitrary finite number $\mathcal{N}$ of elementary cells $\mathcal{F}_{i}$. The transmission coefficient for a finite specimen is proven to be $T=T_{G 22}^{-1}$, where $T_{G 22}$ represents the second diagonal term of the $\mathcal{N}$ th power of $T_{i}$ (see supplementary material). To experimentally prove the properties of the frequency spectra, finite canonical nylon rods with $C=1$ were manufactured, where the elements $L$ and $S$ possess circular cross sections of diameter $40 \mathrm{~mm}$ and $20 \mathrm{~mm}$, respectively. The transmissibility of the rods, defined as $T=20 \log _{10}\left(a_{r} / a_{l}\right)$, was obtained from experiments in which the accelerations of the excited end, $a_{r}$, and the free end, $a_{l}$, of the rod were measured. Details of the experimental setup can be found in the supplementary material.

(a)

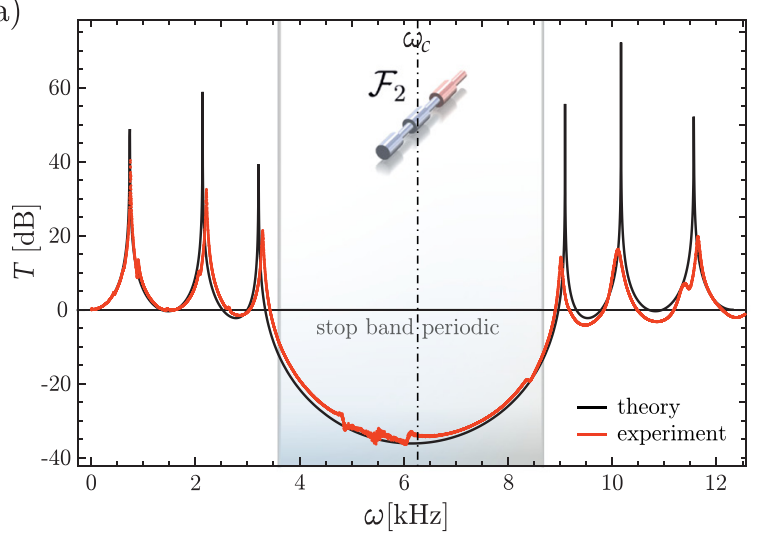

(c)

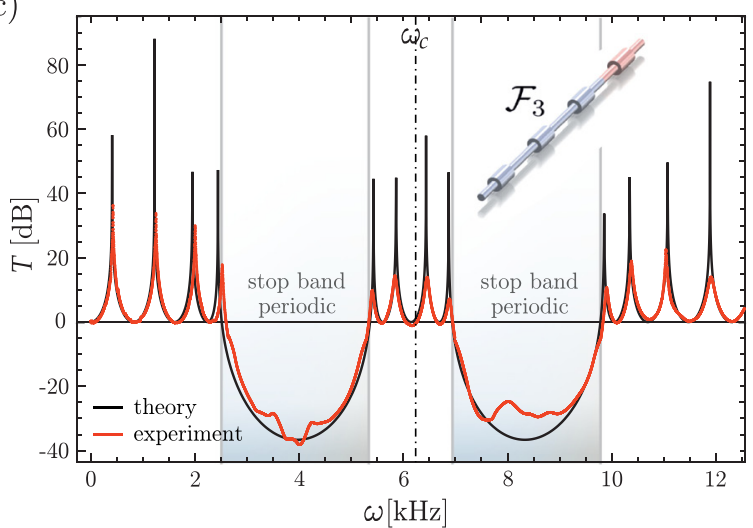

Figure 5 shows the measured transmissibility $T$ of four finite canonical waveguides in excellent agreement with analytical predictions for both the natural frequencies and the regions where wave propagation is significantly attenuated (numerical simulations confirming the agreement are described in the supplementary material). Three configurations, namely, three-cell $\mathcal{F}_{2}$ (a), three-cell $\mathcal{F}_{3}$ (c), and two-cell $\mathcal{F}_{5}(\mathrm{~d})$, present a phase length of $70 \mathrm{~mm}$ and their dynamic spectra, plotted over the first period, result symmetric with respect to the canonical frequency $\omega_{c}$. The three-cell $\mathcal{F}_{2}^{l}$ canonical rod (b), designed with a $140 \mathrm{~mm}$ phase length, shows the periodic response of the frequency spectrum with a period $2 \omega_{c}^{l}$ and the symmetry of the graph of $T$ with respect to the first two canonical frequencies $\omega_{c}^{l}$ and $\omega_{c 1}^{l}$. It should be noted that $\omega_{c}=2 \omega_{c}^{l}$ as the length of each phase for $\mathcal{F}_{2}, \mathcal{F}_{3}$, and $\mathcal{F}_{5}$ is half of that of $\mathcal{F}_{2}^{l}$. On each plot, the stop bands characterizing the infinite periodic waveguides are shown as shaded areas, thus well approximating the regions of negligible wave propagation even for a small number of cells $\mathcal{N}$. Exceptions are represented by limited extension stop bands, Fig. 5(d), which can be detected only with a greater number of unit cells. Finally, the mismatch between the measured and predicted response at high frequencies for $\mathcal{F}_{5}$ is attributed to the large number of interfaces and the viscoelastic behavior of

(b)

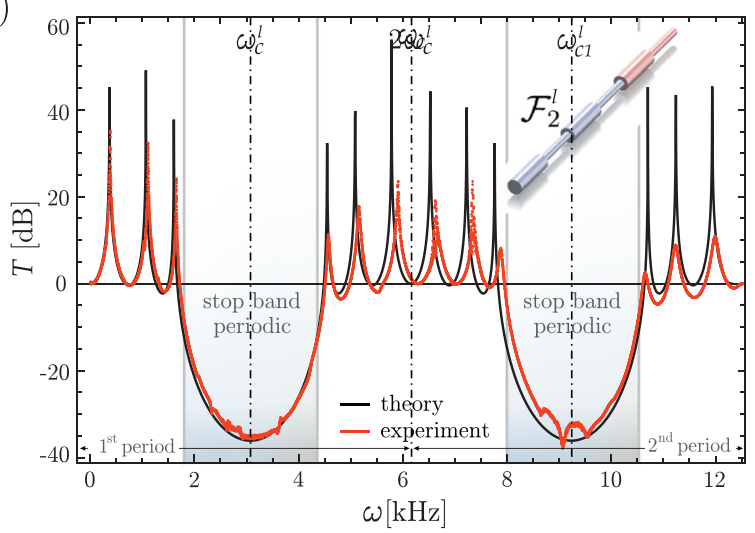

(d)

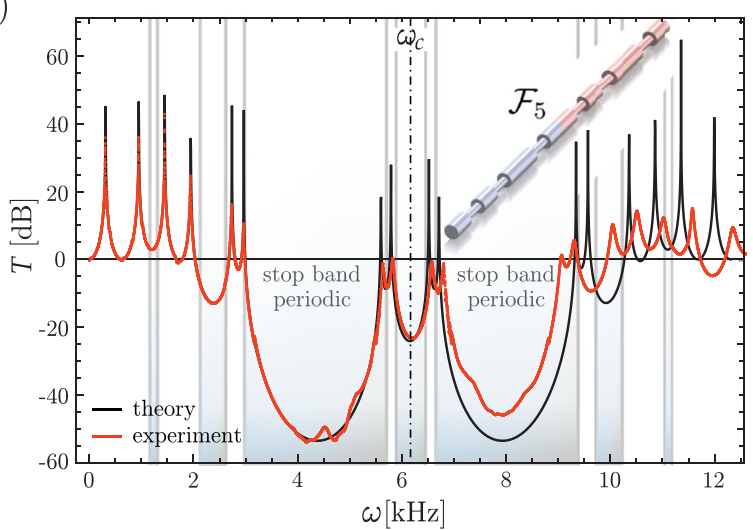

FIG. 5. Comparison between experimental measurements (red line) and analytical predictions (black line) for the transmissibility $T$ of finite canonical waveguides. The response of (a) three-cell $\mathcal{F}_{2}$, (b) three-cell $\mathcal{F}_{2}^{\prime}$, (c) three-cell $\mathcal{F}_{3}$ and (d) two-cell $\mathcal{F}_{5}$ configurations for $\mathrm{C}=1$ is reported as a function of the frequency $\omega$. The stop bands characterizing the infinite periodic waveguides are represented by the underlaid shaded areas. The insets show the geometry of the finite rods, where the red assemblies represent the elementary cells $\mathcal{F}_{i}$. 
the constituent material, also testified by the decrease in the height of the spikes as the frequency increases.

We have shown that there are three families of periodic phononic canonical quasicrystalline-generated waveguides whose Floquet-Bloch spectra are periodic. The period is set by canonical frequencies which geometrically correspond to saddle-point periodic orbits on the invariant manifold. A close inspection of the associated trace map has revealed that there could be multiple periodic orbits at frequencies that differ from the canonical ones. The SPB layout in the neighborhoods of those frequencies is governed by scaling parameters that are expressed in terms of the golden ratio. An experimental verification carried out on finite-size canonical specimens has confirmed the periodicity of the spectra and the extension of stop- and pass-bands. Our research provides new analytical tools for controlling the filtering properties of periodic metamaterials and sets out a methodology that can be extended to waveguides composed of periodic beams, plates, and microarchitected materials.

See the supplementary material for further details on the theory and description of experimental and numerical methods.

M.G. was supported by Italian MIUR under the framework of "Departments of Excellence" Grant L. 232/2016. Z.C. acknowledges support from China Scholarship Council (Grant No. CSC201908500111). L.M. is grateful to the EU's H2020 MSCA COFUND framework under Grant No. 663830. F.B. acknowledges support from the Royal Society (Grant No. RGS-R1-191459).

\section{DATA AVAILABILITY}

The data that support the findings of this study are available from the corresponding author upon reasonable request.

\section{REFERENCES}

'S. Nemat-Nasser, "Harmonic waves in layered composites," J. Appl. Mech. 39, 850-852 (1972).

${ }^{2}$ S. Nemat-Nasser, F. Fu, and S. Minagawa, "Harmonic waves in one-, two-, and three-dimensional composites: Bounds for eigenfrequencies," Int. J. Solids Struct. 11, 617-642 (1975).

${ }^{3}$ M. S. Kushwaha, P. Halevi, L. Dobrzynski, and B. Djafari-Rouhani, "Acoustic band structure of periodic elastic composites," Phys. Rev. Lett. 71, 2022-2025 (1993).

${ }^{4}$ M. M. Sigalas and E. N. Economou, "Elastic and acoustic-wave bandstructure," J. Sound Vib. 158, 377-382 (1992).

${ }^{5} \mathrm{X}$. Zhang and Z. Liu, "Negative refraction of acoustic waves in twodimensional phononic crystals," Appl. Phys. Lett. 85, 341-342 (2004).

${ }^{6}$ B. Morvan, B. Tinel, A.-C. Hladky-Hennion, J. Vasseur, and B. Dubus, "Experimental demonstration of the negative refraction of a transverse elastic wave in a two-dimensional solid phononic crystal," Appl. Phys. Lett. 96, 101905 (2010).

${ }^{7}$ R. Zhu, X. N. Liu, G. K. Hu, C. T. Sun, and G. L. Huang, "Negative refraction of elastic waves at the deep-subwavelength scale in a single-phase metamaterial," Nat. Commun. 5, 5510 (2014).

${ }^{8}$ P. Wang, L. Lu, and K. Bertoldi, "Topological phononic crystals with one-way elastic edge waves," Phys. Rev. Lett. 115, 104302 (2015).

${ }^{9}$ J. Ma, D. Zhou, K. Sun, X. Mao, and S. Gonella, "Edge modes and asymmetric wave transport in topological lattices: Experimental characterization at finite frequencies," Phys. Rev. Lett. 121, 094301 (2018).
${ }^{10}$ M. I. N. Rosa, R. K. Pal, J. R. F. Arruda, and M. Ruzzene, "Edge states and topological pumping in spatially modulated elastic lattices," Phys. Rev. Lett. 123, 034301 (2019).

${ }^{11}$ S. Yang, J. H. Page, Z. Liu, M. L. Cowan, C. T. Chan, and P. Sheng, "Focusing of sound in a 3D phononic crystal," Phys. Rev. Lett. 93, 024301 (2004).

${ }^{12}$ S. Guenneau, A. B. Movchan, G. Petursson, and S. A. Ramakrishna, "Acoustic metamaterials for sound focusing and confinement,” New J. Phys. 9, 399 (2007).

${ }^{13}$ R. E. Vines, J. P. Wolfe, and A. V. Every, "Scanning phononic lattices with ultrasound," Phys. Rev. B 60, 11871-11874 (1999).

${ }^{14} \mathrm{P}$. Wang, J. Shim, and K. Bertoldi, "Effects of geometric and material nonlinearities on tunable band gaps and low-frequency directionality of phononic crystals," Phys. Rev. B 88, 014304 (2013).

${ }^{15}$ E. G. Barnwell, W. J. Parnell, and I. D. Abrahams, "Effects of geometric and material nonlinearities on tunable band gaps and low-frequency directionality of phononic crystals," Extreme Mech. Lett. 12, 23-29 (2017).

${ }^{16}$ D. J. Apigo, K. Qian, E. Prodan, and C. Prodan, "Topological edge modes by smart patterning," Phys. Rev. Mater. 2, 124203 (2018).

${ }^{17}$ D. J. Apigo, W. Cheng, K. F. Dobiszewski, E. Prodan, and C. Prodan, "Observation of topological edge modes in a quasiperiodic acoustic waveguides,” Phys. Rev. Lett. 122, 095501 (2019).

${ }^{18}$ X. Ni, K. Chen, M. Weiner, D. J. Apigo, C. Prodan, A. Alù, E. Prodan, and A. B. Khanikaev, "Observation of topological edge modes in a quasiperiodic acoustic waveguides," Commun. Phys. 2, 55 (2019).

${ }^{19}$ Y. Xia, A. Erturk, and M. Ruzzene, "Topological edge states in quasiperiodic locally resonant metastructures," Phys. Rev. Appl. 13, 014023 (2020).

${ }^{20} \mathrm{M}$. Kolar and F. Nori, "Trace maps of general substitutional sequences," Phys. Rev. B 42, 1062-1065 (1990).

${ }^{21}$ M. Kolar and M. K. Ali, "One-dimensional generalized Fibonacci tilings," Phys. Rev. B 41, 7108-7112 (1990).

${ }^{22} \mathrm{M}$. Baake, U. Grimm, and D. Joseph, "Trace maps, invariants, and some of their applications," Int. J. Mod. Phys. B 07, 1527-1550 (1993).

${ }^{23}$ M. Kohmoto, L. P. Kadanoff, and C. Tang, "Localization problem in one dimension: Mapping and escape," Phys. Rev. Lett. 50, 1870-1872 (1983).

${ }^{24}$ X. Wang, U. Grimm, and M. Schreiber, "Trace and antitrace maps for aperiodic sequences: Extensions and applications," Phys. Rev. B 62, 14020-14031 (2000).

${ }^{25} \mathrm{~L}$. Morini and M. Gei, "Waves in one-dimensional quasicrystalline structures: Dynamical trace mapping, scaling and self-similarity of the spectrum," J. Mech. Phys. Solids 119, 83-103 (2018).

${ }^{26}$ M. Kolar, "New class of one dimensional quasicrystals," Phys. Rev. B 47, 5489-5492 (1993).

${ }^{27}$ The exponent of the element $\mathcal{F}_{i-2}$ of the recursion rule is 1. A larger whole exponent would lead to a sequence for which the function $I(\omega)$ defined in Eq. (4) is no longer an invariant. ${ }^{2}$

${ }^{28}$ Henceforth, the notation $\mathcal{F}_{i}$, where $i$ is the index, will indicate both the sequence and the elementary cell of the structured rod.

${ }^{29} \mathrm{M}$. Gei, "Wave propagation in quasiperiodic structures, stop/pass band distribution and prestress effects," Int. J. Solids Struct. 47, 3067-3075 (2010).

${ }^{30}$ All the theory illustrated in the paper can be equally developed if a periodic mechanical system composed of mass/spring pairs ${ }^{35}$ is analyzed in place of the 1D solid waveguide.

${ }^{31}$ The argument $\omega$ will be dropped from functions $x_{i}(\omega)$ from now on to ease the notation if not strictly necessary.

${ }^{32} \mathrm{M}$. Kohmoto and Y. Oono, "Cantor spectrum for an almost periodic Schroedinger equation and a dynamical map," Phys. Lett 102, 145-148 (1984).

${ }^{33}$ M. Kohmoto, B. Sutherland, and C. Tang, "Critical wave functions and Cantor-set spectrum of a one-dimensional quasicrystal model,” Phys. Rev. B 35, 1020-1033 (1987).

${ }^{34} P_{1}=(0,0, a), \quad P_{2}=(-a, 0,0), \quad P_{3}=(0,-a, 0), \quad P_{4}=(0,0,-a), \quad P_{5}$ $=(a, 0,0), P_{6}=(0, a, 0)$ where $a=\sqrt{4+I(\omega)}$.

${ }^{35}$ M. I. Hussein, M. J. Leamy, and M. Ruzzene, "Dynamics of phononic materials and structures: Historical origins, recent progress, and future outlook," Appl. Mech. Rev. 66, 040802 (2014) 